\title{
Unexpected Potential of Adult Stem Cells
}

\author{
C.M. VERFAILLIE, R. SCHWARTZ, M. REYES, AND Y. JIANG \\ Stem Cell Institute, Department of Medicine, University of Minnesota Medical School, \\ Minneapolis, Minnesota 55455, USA
}

KEYWORDS: multipotent adult progenitor cells; cell differentiation

We have isolated from marrow of humans, mouse, and rat, cells that we have termed multipotent adult progenitor cells or MAPCs. ${ }^{1-5}$ MAPCs appear in mesenchymal stem cell cultures after 25-35 population doublings, provided that cultures are maintained on fibronectin, not collagens or laminin, in the presence of low amounts or no fetal bovine serum, and in the presence of epidermal growth factor and platelet-derived growth factor, supplemented with leukemia inhibitor factor in mouse and rat. In addition, MAPCs are only found when cells are plated and maintained at low density, and are not allowed to grow to semi-confluence or confluence. When maintained under these conditions, MAPCs from $60 \%$ of human bone marrow have been culture-expanded for more than 50 and up to 80 population doublings, and MAPCs from mouse and rat bone marrow have been expanded for more than 80-150 population doublings. Of note, this expansion is not associated with obvious genetic instability, as cytogenetic evaluation of all human and rat cultures, and all but two subpopulations of mouse cultures have not revealed cytogenetic abnormalities, suggesting that this may not be the result of a transforming event. However, the status of most oncogenes and anti-oncogenes has not yet been evaluated. Furthermore, telomeres are apparently stable in mouse, rat, and human MAPC cultures, and telomerase is active.

Differentiation of MAPCs to different lineages is obtained when cells are replated at high density, without the cytokines important for MAPC expansion cultures, and without serum. Using methods originally described by the Caplan group, we can induce osteoblast differentiation (using ascorbic acid, dexamethasone, and $\beta 2$-glycerophosphate), chondroblasts (using transforming growth factor beta-1), adipocytes (using insulin) and skeletal myoblasts (using 5-azacytidin). ${ }^{1,6-8}$ Although MSCs cannot differentiate in endothetial cells, we have shown that MAPCs express the VEGF receptor Flk1, and that addition of VEGF to confluent MAPCinduced sequential acquisition of VE-cadherin and CD34, followed by more mature endothelial markers such as von Willebrand factor (vWF) and platelet endothelium

Address for correspondence: Dr. Catherine Verfaillie, University of Minnesota Medical School, 420 Delaware Street S.E., MC716, Minneaplois, MN 55455. Voice: 612-625-0602; fax: 612-624-2436.

verfa001@tc.umn.edu

Ann. N.Y. Acad. Sci. 996: 231-234 (2003). ( 2003 New York Academy of Sciences. 
cell adhesion molecule (PECAM). ${ }^{4}$ After 14-21 days, more than $80 \%$ of cells acquire endothelial morphology and phenotype, and also have functional characteristics of endothelium including release of vWF upon histamine stimulation, vascular tube formation, and contribution to neo-angiogenesis in vivo.

Aside from differentiation into cells of the mesoderm, we also have evidence that MAPCs can differentiate into cells with neuroectodermal ${ }^{5}$ and endodermal ${ }^{2,5}$ characteristics. When MAPCs are cultured with basic fibroblast growth factor (FGF) followed by the midbrain-specific FGF8 and the neurotrophic factor, brain-derived neurotrophic factor (BDNF), cells acquire morphologic and phenotypic characteristics of neurons, astrocytes, and oligodendrocytes. ${ }^{5}$ During the initial phases of differentiation, expression of transcription factors important in neural differentiation is seen, as well as the neural progenitor-specific nestin gene. After 14-21 days, more than $70 \%$ of cells have died, but remaining cells become polarized and $20 \%$ express dopamine and tyrosine hydroxylase, 20\% express tryptophan hydroxylase and serotonin, whereas the remaining 50\% express gaba-aminobutyric acid (GABA). Preliminary studies using patch clamp examination also demonstrate that neuron-like cells that can be maintained for at least 20-25 days in differentiation culture have electrophysiological characteristics compatible with neurons.

We have also shown that MAPCs can differentiate into hepatocyte-like cells, when cultured with FGF4 and hepatocyte growth factor (HGF). ${ }^{2}$ In FGF4- and HGFcontaining cultures, cells first express cytokeratin (CK)-19 and alpha-fetoprotein $\alpha \mathrm{FP}$ ), and by day 14-21 express CK18, albumin, HepPar-1, and CD26. From day 14-21, cells also acquire functional characteristics of hepatocytes, including secretion of albumin and urea, storage of glycogen, uptake of low-density lipoproteins, and phenobarbital-inducible cytochrome $\mathrm{p} 450$.

To address the question of whether the different lineages are derived from single cells or multiple progenitors present in these bone marrow cultures, we have attempted to clone single cell clones. However, until now this has not been possible. ${ }^{1,5}$ We therefore used retroviral marking to assess whether a single cell can give rise to cells of mesoderm, neuroectoderm, and endoderm, exploiting the fact that retroviruses integrate randomly in the host cell genome, and that DNA sequence flanking the retroviral integrant is unique for each transduced cell and all its progeny. ${ }^{9,10}$ Using Southern blot analysis and flanking region PCR-based sequencing, ${ }^{11}$ we have shown for several human, mouse, and rat populations that a single retroviral integrant could be detected in MAPCs and that the integrant was also present in endothelial, neuroectodermal and hepatocyte-like cells. ${ }^{1,2,5}$

Surprisingly, when mouse MAPCs were injected in the blastocyst, chimerism could be detected in most if not all somatic tissues of up to $80 \%$ of mice derived from blastocysts in which 10-12 cells were injected and 30\% of mice in which a single MAPC was injected, attesting to the multipotency of the MAPC. ${ }^{5}$ Whether MAPCs can also contribute to the germ line is currently being evaluated. When MAPCs were injected intravenously into postnatal murine recipients either without radiation or with sublethal irradiation, engraftment of MAPCs in the hematopoietic system, and in gut, lung, and liver could be seen. Cells present in these organs acquired phenotypic and morphologic characteristics consistent with hematopoietic cells, and epithelial cells, respectively. ${ }^{5}$ Yet to be determined is whether the MAPC-derived progeny can functionally contribute to these organs. Also yet to be formally ruled out is that engraftment seen in the postnatal transplant model, or the chimerism seen 
when MAPCs are injected in the blastocyst may be caused by fusion of MAPCs with organ-specific cells, or with cells of the inner cell mass. However, the high levels of "balanced chimerism" seen in some of the chimeric animals, and the fact that $1-3 \%$ engraftment was seen in non-injured animals, would argue against fusion, although formal studies to fully rule out this possibility are planned. However, as in vitro cultured cells are always euploid, and MAPCs are not co-cultured with endothelia or neural or endodermal cells to induce in vitro differentiation, fusion cannot explain the in vitro differentiation ability of MAPCs to cells of all three embryonic layers.

One of the major questions that remains unanswered is whether MAPCs exist as such in vivo or whether this phenotype is induced in vitro. There are arguments to support either notion, although none of them are conclusive, and studies are ongoing to further evaluate this question. The finding that 35-35 population doublings are required for the multipotent nature of MAPCs to become apparent could suggest that MAPCs, which are rare, survive better under the culture conditions used than MSCs and that it therefore requires $>30$ PDs for the majority of cells in culture to be MAPCs. The same finding can also be used to suggest that it takes a certain period of time to induce the MAPC phenotype in MSCs. It is interesting to note that we have also isolated MAPCs from mouse brain tissue and mouse muscle tissue. The phenotype and expressed gene profile of MAPCs generated from these cultures, as well as their ability to differentiate, are highly similar to those of marrow-derived MAPCs. ${ }^{3}$ As there are no obvious genes in the cDNA analysis studies that link brain-derived MAPCs to neuroectodermal cells or muscle-derived MAPCs to myoblasts, one could argue that MAPCs must exist in multiple organs, and that no "reprogramming" is required to elicit the phenotype. However, as we did not use highly purified tissues, but all cells within brain and muscle, it is possible that the same cell in bone marrow that can be induced to acquire the MAPC phenotype circulates and can be harvested from other organs, or that MSCs in brain or muscle can, like their counterparts in marrow, be induced to acquire a MAPC phenotype. Prospective isolation experiments will be needed to determine whether MAPCs exist in vivo. This question is not only of academic or pure basic science interest, but may have far-reaching, implications for the ultimate usefulness of MAPCs. If the phenotype is induced in vitro, insights gained in the mechanism underlying this reprogramming event should yield improved culture systems, and eventually potential specific molecular triggers that can induce such apparent de-differentiation with greater precision and frequency. On the other hand, if the cells exist in vivo, important experiments to be done will be studies aimed at testing the exact role of MAPCs in vivo and their ability to proliferate in vivo and possibly to be mobilized to tissues or organs that are damaged, and directed to differentiate in those tissues.

\section{REFERENCES}

1. Reyes, M., T. Lund, T. LenviK, et al. 2001. Purification and ex vivo expansion of postnatal human marrow mesodermal progenitor cells. Blood 98: 2615-2625.

2. Schwartz, R.E., M. Reyes, L. Koodie, et al. 2002. Multipotent adult progenitor cells from bone marrow differentiate into functional hepatocyte-like cells. J. Clin. Invest. 96: $1281-1302$.

3. JiAnG, Y., B. VAESSEN, T. LeNviK, et al. 2002. Multipotent progenitor cells can be isolated from post-natal murine bone marrow, muscle and brain. Exp. Hematol. In press.

4. Reyes, M., A. Dudek, B. Jahagirdar, et al. 2002. Origin of endothelial progenitors in human past-natal bane marrow. J. Clin. Invest. 109: 337-346. 
5. JiAng, Y., B. JAhAgirdar, M. Reyes, et al. 2002. Pluripotent nature of adult marrowderived mesenchymal stem cells. Nature 418: 41-49.

6. Haynesworth, S.E., M.A. Barber \& I.M. Caplan. 1992. Cell surface antigens on human marrow-derived mesenchymal cells are detected by monoclonal antibodies. Bone 13: 69-80.

7. Haynesworth, S., M. Baber \& A. Caplan. 1996. Cytokine expression by human marrow-derived mesenchymal progenitor cells in vitro: effects of dexamethasone and IL-1 alpha. J. Cell Physiol. 166: 585-592.

8. Wakitani, S., T. Saito \& A. Caplan. 1995. Myogenic cells derived from rat bone marrow mesenchymal stem cells exposed to 5-azacytidine. Muscle Nerve 18: 1417 1426.

9. Jordan, C., J. McKearn \& I. LemischKa. 1990. Cellular and developmental properties of fetal hematopoietic stem cells. Cell 61: 953-963.

10. Nolta, J., M. DaO, S. Wells, et al. 1996. Transduction of pluripotent human hematopoietic stem cells demonstrated by clonal analysis after engraftment in immune-deficient mice. Proc. Natl. Acad. Sci. USA 93: 2414-2419.

11. LeNVIK, T., T. Lund \& C.M. Verfaillie. 2002. Blockerette-ligated catpture T7 amplified RT-PCR: a new method for determining flankin sequences. Mol. Therapy 6: $113-1188$. 